\title{
DESIGN OF THE STATE PREDICTIVE MODEL FOLLOWING CONTROL SYSTEM WITH TIME-DELAY
}

\author{
DAZHONG WANG，SHUJING WU，SHIGENORI OKUBO \\ Faculty of Engineering \\ Yamagata University, Jonan, Yonezawa,Yamagata, Japan \\ e-mail: wdzh168@hotmail.com
}

\begin{abstract}
Time-delay systems exist in many engineering fields such as transportation systems, communication systems, process engineering and, more recently, networked control systems. It usually results in unsatisfactory performance and is frequently a source of instability, so the control of time-delay systems is practically important. In this paper, a design of the state predictive model following control system (PMFCS) with time-delay is discussed. The bounded property of the internal states for the control is given, and the utility of this control design is guaranteed. Finally, examples are given to illustrate the effectiveness of the proposed method, and state predictive control techniques are applied to congestion control synthesis problems for a TCP/AQM network.
\end{abstract}

Keywords: state predictive control, time-delay, model following control system (MFCS), TCP/AQM network, congestion control.

\section{Introduction}

Communication systems, transmission systems, chemical processing systems, metallurgical processing systems, environmental systems and power systems are all examples of time-delay systems (Wood and Berry, 1973; Alevisakis and Seborg, 1974). In recent years, such systems have attracted recurring interest of research community. Much of the research work has been focused on stability analysis and the stabilization of time-delay systems using the so-called Lyapunov-Krasovskii functional and the linear matrix inequality (LMI) approach (Zhang and Xie, 2007). The model following control system (MFCS) method is one of the most promising ways of handling control problem for systems having input or state constraints (Okubo, $1985 ;$ 1992). One way to cope with such problems is to develop a time delay MFCS method (Akiyama et al., 1998). This paper is dedicated to the design of the state predictive model following control system (PMFCS) with input time-delay. The design of the control system is performed using an easy algebraic algorithm of matrices whose elements are polynomials of the operator. The bounded property of internal states for the control system is given and the utility of this control design is guaranteed. It is confirmed on the basis of Example 1 that the output of the control system asymptotically follows the reference model in the case of the existence of disturbances.

Time-delay is very important for the modeling of networks, occurring both in the control of networks and in the control over networks (Chiasson, 2007). In the context of communication networks, the term "congestion control" is generally used to refer to the action of regulating various flows within a network. In recent years, intense research efforts have been devoted to the application of the Smith predictor for queue length control of ATM (asynchronous transfer mode) networks (Mascolo, 2000). The transmission control protocol (TCP) was designed in the late 1980s by Van Jacobson (Chiasson, 2007), which is a critical part of the Internet machinery. TCP/AQM (transmission control protocol/ active queue management) networks, which consist of TCP networks and AQM mechanisms (congestion controllers), are considered and dynamical models of the average TCP window size and the queue size in the bottleneck router are derived. Since dynamical models are nonlinear, the models are linearized at an equilibrium point and PI congestion controllers are designed. But linearized models depend on information delay, which is called time-delay, and it is difficult to design controllers which are assured to have better stability and performance for models with time delay $(\mathrm{Gu}$ et al., 2007; Hollot et al., 2001a; 2002; 2001b; Misra et al., 2000; Azuma et al., 2005a; 2005b; 2005c). 
In this paper, state predictive control techniques are applied to congestion control synthesis problems for a TCP/AQM network. State predictive control uses directly time delay for an input time delay system and the system can be transformed into a linear time invariant one by using a variable transformation. Thus it is easy to design controllers with enough stability and performance based on linear MFCS theory. We have verified the methods with Example 2 and therefore found that the system was stable and applicable.

The organization of this paper is as follows: In the next section, the controlled object and the reference model are described. Section 3 focuses on the state predictive control system with time-delay. The design of the state predictive model following control system with timedelay is then described in Section 4. In Section 5, the calculated characteristic polynomial $\left|p E_{s}-A_{s}\right|$ proves the bounded property of the internal states. Some simulations are reported (Examples 1 and 2) in Section 6. Finally, Section 7 concludes the paper.

\section{Notation}

For convenience, we define some notation that will be used throughout this paper as follows:

$\mathbb{R}, \mathbb{R}^{n}, \mathbb{R}^{m \times n}:$ the sets of real numbers, vectors and matrices of specified dimensions.

$I$ : the identity matrix.

$A^{-1}$ : the inverse of the matrix $A$.

$|A|$ : the determinant of the matrix $A$.

\section{Problem statement}

The linear system with time-delay and disturbances is described by the following state equations:

$$
\begin{aligned}
& \dot{x}(t)=A x(t)+B u(t-L)+d(t), \\
& y(t)=C x(t)+d_{0}(t),
\end{aligned}
$$

where $A \in \mathbb{R}^{n \times n}, B \in \mathbb{R}^{n \times l}, C \in \mathbb{R}^{l \times n} ; x(t) \in \mathbb{R}^{n}$, $u(t) \in \mathbb{R}^{l}, y(t) \in \mathbb{R}^{l}$ are the internal state vector whose elements are available, the control input vector and the available states output vector of the system; $d(t) \in \mathbb{R}^{n}$ and $d_{0}(t) \in \mathbb{R}^{l}$ are bounded disturbances; $t$ is the time, $L \geq 0$ is the time-delay.

Assumption 1 Assume that $[A, B]$ is controllable and $[C, A]$ is observable, i.e., the following conditions hold:

$$
\begin{aligned}
\operatorname{rank}\left[\begin{array}{cc}
p I-A, B & -A
\end{array}=n,\right. \\
\operatorname{rank}\left[\begin{array}{c}
p I-A \\
C
\end{array}\right]=n .
\end{aligned}
$$

Assumption 2 In order to guarantee the existence and uniqueness of the solution and to have an exponential function mode but an impulse one for (1) and (2), the following condition is assumed:

$$
|p I-A| \not \equiv 0 .
$$

Assumption 3 Zeros of $C[p I-A]^{-1} B$ are stable.

The reference model is given below, which is assumed controllable and observable:

$$
\begin{aligned}
& \dot{x}_{m}(t)=A_{m} x_{m}(t)+B_{m} r_{m}(t), \\
& y_{m}(t)=C_{m} x_{m}(t) .
\end{aligned}
$$

Here $y_{m}(t) \in \mathbb{R}^{l_{m}}, r_{m}(t) \in \mathbb{R}^{l_{m}}, x_{m}(t) \in \mathbb{R}^{n_{m}} \cdot r_{m}(t)$ is the model input, $y_{m}(t)$ is the model output. $A_{m} \in$ $\mathbb{R}^{n_{m} \times n_{m}}, B_{m} \in \mathbb{R}^{n_{m} \times l_{m}}, C_{m} \in \mathbb{R}^{l_{m} \times n_{m}}$

The output error is given as

$$
e(t)=y(t)-y_{m}(t) .
$$

The aim of control system design is to obtain a control law which makes the output converge to a zero error and keeps the internal states bounded.

\section{State predictive control system with time- delay}

Assume that $\hat{x}(t)=x(t+L)$ is the state predictive signal. Then $\hat{x}(t)$ can be rewritten as follows (Watanabe and Ito, 1981; Kim and Bien, 1981):

$$
\begin{aligned}
\hat{x}(t)= & e^{A L} x(t)+\int_{t-L}^{t} e^{A(t-\tau)} B u(\tau) \mathrm{d} \tau \\
& +\int_{t-L}^{t} e^{A(t-\tau)} d(\tau+L) \mathrm{d} \tau .
\end{aligned}
$$

Then, we have

$$
\begin{aligned}
\dot{\hat{x}}(t)= & \dot{x}(t+L)=e^{A L} A x(t) \\
& +A \int_{t-L}^{t} e^{A(t-\tau)} B u(\tau) \mathrm{d} \tau+B u(t) \\
& +A \int_{t-L}^{t} e^{A(t-\tau)} d(\tau+L) \mathrm{d} \tau+d(t+L) .
\end{aligned}
$$

The state equations of (1) and (2) can be rewritten as

$$
\begin{aligned}
& \dot{\hat{x}}(t)=A \hat{x}(t)+B u(t)+\hat{d}(t), \\
& \hat{y}(t)=C \hat{x}(t)+\hat{d}_{0}(t),
\end{aligned}
$$

where $\hat{d}(t)=d(t+L), \hat{d}_{0}(t)=d_{0}(t+L), \hat{y}(t)=y(t+L)$. The reference model for state predictive is given by

$$
\begin{aligned}
& \dot{\hat{x}}_{m}(t)=A_{m} \hat{x}_{m}(t)+B_{m} \hat{r}_{m}(t), \\
& \hat{y}_{m}(t)=C_{m} \hat{x}_{m}(t),
\end{aligned}
$$

where $\hat{x}_{m}(t)=x_{m}(t+L), \hat{y}_{m}(t)=y_{m}(t+L), \hat{r}_{m}(t)=$ $r_{m}(t+L)$.

The state predictive output error (5) is given as

$$
\hat{e}(t)=\hat{y}(t)-\hat{y}_{m}(t) .
$$

Here, $\hat{e}=e(t+L)$. 


\section{Design of the state predictive model following control system with time-delay}

Letting $p=\mathrm{d} / \mathrm{d} t$, from (6) and (8), $\hat{x}(t)$ and $\hat{x}_{m}(t)$ can be rewritten as

$$
\begin{aligned}
\hat{x}(t) & =[p I-A]^{-1} B u(t)+[p I-A]^{-1} \hat{d}(t), \\
\hat{x}_{m}(t) & =\left[p I-A_{m}\right]^{-1} B_{m} \hat{r}_{m}(t) .
\end{aligned}
$$

Using (7), (9), (11) and (12), we have

$$
\begin{aligned}
\hat{y}(t)= & C[p I-A]^{-1} B u(t) \\
& +C[p I-A]^{-1} \hat{d}(t)+\hat{d}_{0}(t), \\
\hat{y}_{m}(t)= & C_{m}\left[p I-A_{m}\right]^{-1} B_{m} \hat{r}_{m}(t) .
\end{aligned}
$$

Then the representations of input-output equations are given as

$$
\begin{aligned}
D(p) \hat{y}(t) & =N(p) u(t)+\hat{w}(t), \\
D_{m}(p) \hat{y}_{m}(t) & =N_{m}(p) \hat{r}_{m}(t),
\end{aligned}
$$

where $C[p I-A]^{-1} B=N(p) / D(p), N(p)=C \operatorname{adj}[p I-$ $A] B \in R^{l \times l}, D(p)=|p I-A|, C_{m}\left[p I-A_{m}\right]^{-1} B_{m}=$ $N_{m}(p) / D_{m}(p), N_{m}(p)=C_{m} \operatorname{adj}\left[p I-A_{m}\right] B_{m} \in$ $\mathbb{R}^{l_{m} \times l_{m}}$ and $D_{m}(p)=\left|p I-A_{m}\right|$. The disturbance $\hat{w}(t)$ is thus obtained:

$$
\hat{w}(t)=C \operatorname{adj}[p I-A] \hat{d}(t)+D(p) \hat{d}_{0}(t) .
$$

Then $N(p)$ and $N_{m}(p)$ are given by

$$
\begin{aligned}
N(p) & =\operatorname{diag}\left(p^{\eta_{i}}\right) N_{r}+\tilde{N}(p), \\
N_{m}(p) & =\operatorname{diag}\left(p^{\eta_{m_{i}}}\right) N_{m_{r}}+\tilde{N}_{m}(p) . r
\end{aligned}
$$

Here, $\partial_{r_{i}} \tilde{N}(p)<\eta_{i} \partial_{r_{i}} \tilde{N}_{m}(p)<\eta_{m_{i}}, \partial_{r_{i}}(\cdot)$ is the minimum degree of $(\cdot) . N_{r}$ is fixed matrix of $l \times l$, and $\left|N_{r}\right| \neq 0$. The disturbances $d(t)$ and $d_{0}(t)$ are bounded and satisfy

$$
D_{d}(p) \hat{d}(t)=0, D_{d}(p) \hat{d}_{0}(t)=0 .
$$

Here, $D_{d}(p)$ is a scalar characteristic polynomial of disturbances. From (14), we have

$$
D_{d}(p) \hat{w}(t)=0 .
$$

Choose a stable polynomial $T(p)$ which satisfies the following conditions:

(i) The degree of $T(p)$ is $\rho \geq n_{d}+2 n-n_{m}-1-\eta_{i}$.

(ii) The coefficient of the maximum degree term of $T(p)$ is the same as $D(p)$.

Consider the following equation:

$$
T(p) D_{m}(p)=D_{d}(p) D(p) R(p)+S(p),
$$

where the degree of each polynomial is $\partial T(p)=\rho$, $\partial D_{m}(p)=n_{m}, \partial D_{d}(p)=n_{d}, \partial D(p)=n, \partial R(p)=$ $\rho+n_{m}-n_{d}-n, \partial S(p) \leq n_{d}+n-1 T(p), D_{m}(p)$, $D_{d}(p), D(p), R(p)$ are nomic polynomials. Then the following form is obtained:

$$
\begin{aligned}
T(p) D_{m}(p) \hat{e}(t)= & D_{d}(p) D(p) R(p) \hat{y}(t) \\
& +S(p) \hat{y}(t)-T(p) N_{m}(p) \hat{r}_{m}(t) .
\end{aligned}
$$

Namely,

$$
\begin{aligned}
T(p) D_{m}(p) \hat{e}(t)= & D_{d}(p) R(p)(N(p) u(t)+\hat{w}(t)) \\
& +S(p) \hat{y}(t)-T(p) N_{m}(p) \hat{r}_{m}(t) \\
= & D_{d}(p) R(p) N(p) u(t)+S(p) \hat{y}(t) \\
& -T(p) N_{m}(p) \hat{r}_{m}(t) \\
= & {\left[D_{d}(p) R(p) N(p)-Q(p) N_{r}\right] u(t) } \\
& +Q(p) N_{r} u(t)+S(p) \hat{y}(t) \\
& -T(p) N_{m}(p) \hat{r}_{m}(t)
\end{aligned}
$$

where

$$
Q(p)=\operatorname{diag}\left(p^{\rho+n_{m}-n+\eta_{i}}\right)+\widetilde{Q}(p), \quad i=1,2, \ldots, l,
$$

$\widetilde{Q}(p)$ is a polynomial matrix which is stable and $\partial_{r i} \widetilde{Q}(p)<\rho+n_{m}-n+\eta_{i}$. The next control law $u(t)$ can be obtained by making the right-hand side of (17) equal to zero. Thus,

$$
\begin{aligned}
u(t)= & -N_{r}^{-1} Q^{-1}(p)\left\{D_{d}(p) R(p) N(p)\right. \\
& -Q(p) N_{r} u(t) \\
& -N_{r}^{-1} Q^{-1}(p) S(p) \hat{y}(t)+u_{m}(t), \\
u_{m}(t)= & N_{r}^{-1} Q^{-1}(p) T(p) N_{m}(p) \hat{r}_{m}(t) .
\end{aligned}
$$

To avoid using derivatives of signals in control input $u(t)$, next constraints of polynomial degree must be satisfied.

$$
\begin{aligned}
n_{m}-\eta_{m_{i}} & \geq n-\eta_{i}, & & i=1,2, \ldots, l, \\
\rho & \geq n_{d}+2 n-n_{m}-1-\eta_{i}, & i & =1,2, \ldots, l .
\end{aligned}
$$

The state space expression of $u(t)$ can be given as follows:

$$
\begin{aligned}
u(t)= & -H_{1} \xi_{1}(t)-E_{2} \hat{y}(t)-H_{2} \xi_{2}(t) \\
& +u_{m}(t), \\
u_{m}(t)= & E_{3} \hat{r}_{m}(t)+H_{3} \xi_{3}(t) .
\end{aligned}
$$

The following must be satisfied:

$$
\begin{aligned}
& \dot{\xi}_{1}(t)=F_{1} \xi_{1}(t)+G_{1} u(t), \\
& \dot{\xi}_{2}(t)=F_{2} \xi_{2}(t)+G_{2} \hat{y}(t), \\
& \dot{\xi}_{3}(t)=F_{3} \xi_{3}(t)+G_{3} \hat{r}_{m}(t) .
\end{aligned}
$$




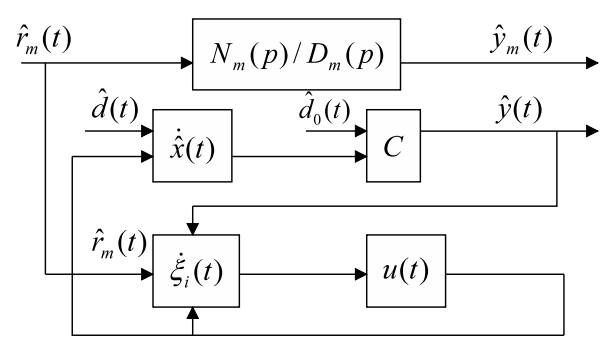

Fig. 1. Block diagram of the state predictive MFCS with timedelay.

Note that there are connections between the polynomial matrices and the system matrices:

$$
\begin{aligned}
H_{1}\left[p I-F_{1}\right]^{-1} G_{1}= & N_{r}^{-1} Q^{-1}(p) D_{d}(p) R(p) \\
& N(p)-Q(p) N_{r}, \\
E_{2}+H_{2}\left[p I-F_{2}\right]^{-1} G_{2}= & N_{r}^{-1} Q^{-1}(p) S(p), \\
E_{3}+H_{3}\left[p I-F_{3}\right]^{-1} G_{3}= & N_{r}^{-1} Q^{-1}(p) T(p) N_{m}(p) .
\end{aligned}
$$

Here, $\left|p I-F_{i}\right|=|Q(p)|(i=1,2,3)$. Therefore, $u(t)$ of (18) is obtained from $\hat{e}(t)=0$. The model following control system can be realized if the system internal states are bounded.

We show a block diagram of the state predictive model following control system with time-delay in Fig. 1.

\section{Bounded property of internal states}

System inputs are the reference input signal $\hat{r}_{m}(t)$ and the disturbances $\hat{d}(t), \hat{d}_{0}(t)$, which are all assumed to be bounded.

Let $z_{s}(t)$ be as follows:

$$
z_{s}(t)=\left[\hat{x}^{T}(t), \xi_{1}^{T}(t), \xi_{2}^{T}(t), u^{T}(t)\right]^{T} .
$$

Remove $u(t)$ from (6), (7), (18) and (22)-(24). Then the system is defined by

$$
\begin{aligned}
E_{s} \dot{z}_{s}(t) & =A_{s} z_{s}(t)+d_{s}(t), \\
y(t) & =C_{s} z_{s}(t)+d_{s 0}(t),
\end{aligned}
$$

where

$$
E_{s}=\left[\begin{array}{cccc}
I & 0 & 0 & 0 \\
0 & I & 0 & 0 \\
0 & 0 & I & 0 \\
0 & 0 & 0 & 0
\end{array}\right],
$$

$$
\begin{aligned}
A_{s} & =\left[\begin{array}{cccc}
A & 0 & 0 & B \\
0 & F_{1} & 0 & G_{1} \\
G_{2} C & 0 & F_{2} & 0 \\
-E_{2} C & -H_{1} & -H_{2} & -I
\end{array}\right], \\
d_{s}(t) & =\left[\begin{array}{c}
d(t) \\
0 \\
G_{2} \hat{d}_{0}(t) \\
-E_{2} \hat{d}_{0}(t)+u_{m}(t)
\end{array}\right], \\
C_{s} & =\left[\begin{array}{llll}
C & 0 & 0 & 0
\end{array}\right], \quad d_{s 0}(t)=\hat{d}_{0}(t) .
\end{aligned}
$$

In (24), $\xi_{3}(t)$ is bounded, because $\left|p I-F_{3}\right|=|Q(p)|$ is a stable polynomial and $\hat{r}_{m}(t)$ is the reference input. The bounded property of internal states turns out to be that $z_{s}(t)$ is bounded. The characteristic polynomial $\mid p E_{s}-$ $A_{s} \mid$ can be calculated as

$$
\left|p E_{s}-A_{s}\right|=T(p)^{l} D_{m}(p)^{l}|Q(p)|\left|N_{r}\right|^{-1} \frac{|N(p)|}{D(p)^{l-1}} .
$$

Because the system is observable and controllable, when exponents of input and output were equal, reduced factorization of the transfer function was as follows:

$$
C[p I-A]^{-1} B=N(p) / D(p)=U(p)^{-1} V(p),
$$

where $V(p)$ is the zero polynomial. Thus

$$
|N(p)|=D(p)^{l-1}|V(p)| .
$$

Furthermore,

$$
\begin{aligned}
\mid p E_{s} & -A_{s} \mid \\
& =\left|N_{r}\right|^{-1} T(p)^{l} D_{m}(p)^{l}|Q(p)||V(p)|
\end{aligned}
$$

with stable polynomials of $\left|N_{r}\right|^{-1}, T(p), D_{m}(p),|Q(p)|$ and $|V(p)|$. Therefore, $A_{s}$ is also a stable system matrix.

Assumption 4 For the controlled system (29) and (30), the rank of $E_{s}$ and the degree about $p$ of $\left|p E_{s}-A_{s}\right|$ satisfy

$$
\begin{aligned}
\operatorname{rank} E_{s} & =\operatorname{deg}\left|p E_{s}-A_{s}\right| \\
& =n+2 \sum_{i=1}^{l}\left(\rho+n_{m}-n+\eta_{i}\right) .
\end{aligned}
$$

In general, the above main results are summarized in the next theorem.

Theorem 1. With the controlled system (1), (2) and the reference model (3), (4), all the internal states are bounded and the output error $e(t)=y(t)-y_{m}(t)$ is asymptotically to converge to zero in the design of the state predictive model following control system with time-delay if the following conditions are held:

1. $\operatorname{rank} E_{s}=\operatorname{deg}\left|p E_{s}-A_{s}\right|$.

2. Zeros of $C[p I-A]^{-1} B$ are stable.

3. $\left|N_{r}\right| \neq 0$. 


\section{Simulation study}

Example 1. In this example, the time-delay is $L=1$, and the disturbances $d(t)$ and $d_{0}(t)$ are step and ramp disturbances, respectively. Then $d(t)$ and $d_{0}(t)$ are given as $d(t)=0.9(6 \leq t \leq 18)$ and $d_{0}(t)=0.03 t-0.5(28 \leq$ $t \leq 50)$. The initial value functions are $x_{0}(t)=[1,-3]^{T}$, $u_{0}(t)=0(t<0)$ and $\xi_{i}^{0}(t)=[0,0]^{T}(i=1,2)$.

The reference model is given by

$$
\begin{aligned}
& \dot{x}_{m}(t)=\left[\begin{array}{cc}
0 & 1 \\
-6 & -5
\end{array}\right] x_{m}(t)+\left[\begin{array}{l}
0 \\
1
\end{array}\right] r_{m}(t), \\
& y_{m}(t)=\left[\begin{array}{ll}
2 & 1
\end{array}\right] x_{m}(t), \\
& r_{m}(t)= \begin{cases}0, & -L \leq t<0, \\
4 \sin (0.5 t)+8, & t \geq 0,\end{cases} \\
& x_{m}(0)=\xi_{3}(0)=[0,0]^{T},
\end{aligned}
$$

for the system defined by

$$
\begin{aligned}
\dot{x}(t)= & {\left[\begin{array}{cc}
0 & 1 \\
-3 & -4
\end{array}\right] x(t)+\left[\begin{array}{l}
0 \\
2
\end{array}\right] u(t-L) } \\
& +\left[\begin{array}{c}
0 \\
d(t)
\end{array}\right], \\
y(t)= & {\left[\begin{array}{ll}
5 & 1
\end{array}\right] x(t)+d_{0}(t) . }
\end{aligned}
$$

From (6) and (7), the system (33) and (34) is given by

$$
\begin{aligned}
& \dot{\hat{x}}(t)= {\left[\begin{array}{cc}
0 & 1 \\
-3 & -4
\end{array}\right] \hat{x}(t)+\left[\begin{array}{l}
0 \\
2
\end{array}\right] u(t) } \\
&+\left[\begin{array}{c}
0 \\
\hat{d}(t)
\end{array}\right] \\
& \hat{y}(t)=\left[\begin{array}{ll}
5 & 1
\end{array}\right] \hat{x}(t)+\hat{d}_{0}(t) .
\end{aligned}
$$

The control input $u(t)$ is given as

$$
\begin{aligned}
u(t)= & {\left[\begin{array}{ll}
36 & 7
\end{array}\right] \xi_{1}(t)-3 \hat{y}(t) } \\
& +\left[\begin{array}{ll}
93 & 22
\end{array}\right] \xi_{2}(t)+u_{m}(t), \\
u_{m}(t)= & 0.5 \hat{r}_{m}(t)-\left[\begin{array}{ll}
13 & 2.5
\end{array}\right] \xi_{3}(t) .
\end{aligned}
$$

Namely,

$$
\begin{aligned}
u(t)= & {\left[\begin{array}{ll}
36 & 7
\end{array}\right] \xi_{1}(t)-3 y(t+L) } \\
& +\left[\begin{array}{ll}
93 & 22
\end{array}\right] \xi_{2}(t)+u_{m}(t), \\
u_{m}(t)= & 0.5 r_{m}(t+L)-\left[\begin{array}{ll}
13 & 2.5
\end{array}\right] \xi_{3}(t) .
\end{aligned}
$$

where

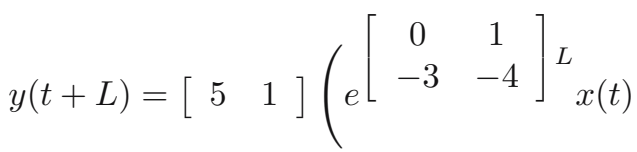

$$
\begin{aligned}
& +\int_{t-L}^{t} e^{\left[\begin{array}{cc}
0 & 1 \\
-3 & -4
\end{array}\right]{ }^{(t-\tau)}}\left[\begin{array}{l}
0 \\
2
\end{array}\right] u(\tau) \mathrm{d} \tau
\end{aligned}
$$

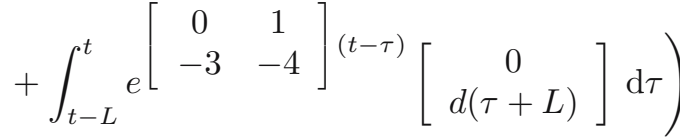

$$
\begin{aligned}
& +d_{0}(t+L) \text {. }
\end{aligned}
$$

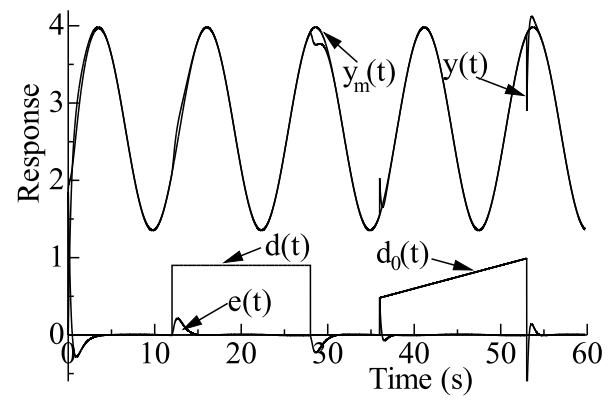

Fig. 2. Responses of the system with time-delay.

We show a result of simulation in Fig. 2. It can be concluded that the output signal follows the reference even if disturbances exist in the system.

Example 2. Hollot et al. (2002) proposed nonlinear mathematic models which describe behaviors of the average window size in senders and the queue size in the bottleneck router in the network are given as follows (Misra et al., 2000; Hollot et al., 2002):

$$
\begin{aligned}
\dot{W}(t) & =\frac{1}{R(t)}-\frac{W(t)}{2} \frac{W(t-R(t))}{R(t-R(t))} \bar{p}(t-R(t)), \\
\dot{\bar{q}}(t) & =\frac{W(t)}{R(t)} N(t)-D(t) \\
R(t) & =\frac{\bar{q}(t)}{D(t)}+T_{p}
\end{aligned}
$$

where $W(t)$ is the congestion window size in packets at time $t, \bar{q}(t)$ is the queue length at the congested router in packets, $R(t)$ is the round trip time (RTT) which represents the delay in TCP dynamics in seconds, $D(t)$ is the link capacity in packets per second, $T_{p}$ is the propagation delay in seconds, $N(t)$ is the number of active TCP connections, $\bar{p}(t)$ is the packet mark/drop probability. Taking $(W, \bar{q})$ as the state and $\bar{p}$ as the input and assuming $N(t)=N, R(t)=R_{0}, D(t)=D, \bar{q}(t)=\bar{q}$ and $W \gg 1$, we define the set of operating points $\left(W_{0}, \bar{q}_{0}, \bar{p}_{0}\right)$ by $\dot{W}=0$ and $\dot{\bar{q}}=0$. Linearize the above mathematical model near the equilibrium point, and use the Laplace transformation. $\delta W \doteq W-W_{0}, \delta \bar{q} \doteq \bar{q}-\bar{q}_{0}$ and $\delta \bar{p} \doteq \bar{p}-\bar{p}_{0}$ are the state variables and input perturbations around the operating point. The control system block diagram (Fig. 3) and the control system are obtained as fol- 


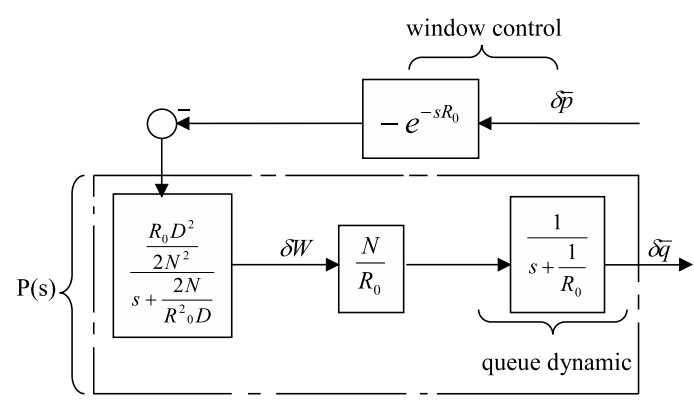

Fig. 3. Block diagram of the congestion control system when $W_{0} \gg 1$.

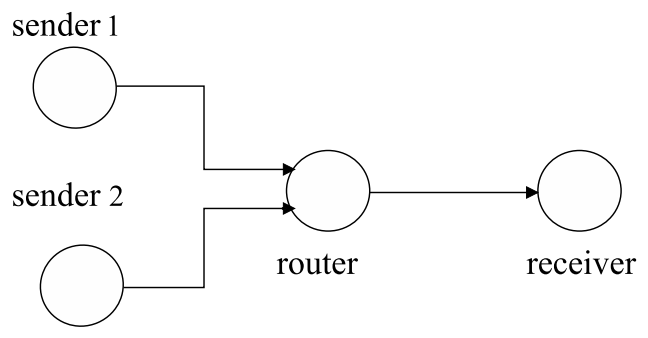

Fig. 4. Testbed network topology.

lows (Hollot et al., 2001 a; 2001b):

$$
\begin{aligned}
\delta \dot{W}(t) & =-\frac{2 N}{R_{0}^{2} D} \delta W(t)-\frac{R_{0} D^{2}}{2 N^{2}} \delta \bar{p}\left(t-R_{0}\right), \\
\dot{\delta} \bar{q}(t) & =\frac{N}{R_{0}} \delta W(t)-\frac{1}{R_{0}} \delta \bar{q}(t), \\
R_{0} & =\frac{\bar{q}_{0}}{D}+T_{p} .
\end{aligned}
$$

The state vector, the output vector and the input vector are introduced as follows:

$x(t)=\left[\begin{array}{c}\delta W(t) \\ \delta \bar{q}(t)\end{array}\right], \quad y(t)=\delta \bar{q}(t), \quad u(t)=\delta \bar{p}(t)$.

The TCP/AQM networks (35) and (36) can be described as the following linear system with information time-delay (Azuma et al., 2005a; 2005b; 2005c):

$$
\begin{aligned}
& \dot{x}(t)=A x(t)+B u\left(t-R_{0}\right), \\
& y(t)=C x(t),
\end{aligned}
$$

where

$$
\begin{aligned}
& A=\left[\begin{array}{cc}
-\frac{2 N}{R_{0}^{2} D} & 0 \\
\frac{N}{R_{0}} & -\frac{1}{R_{0}}
\end{array}\right], \quad B=\left[\begin{array}{c}
-\frac{R_{0} D^{2}}{2 N^{2}} \\
0
\end{array}\right], \\
& C=\left[\begin{array}{ll}
0 & 1
\end{array}\right] .
\end{aligned}
$$

$P(s)$ is defined by (Hollot et al., 2001a; 2001b; Azuma et al., 2005a; 2005b; 2005c)

$$
P(s)=\frac{\frac{D^{2}}{2 N}}{\left(s+\frac{2 N}{R_{0}^{2} D}\right)\left(s+\frac{1}{R_{0}}\right)} .
$$

From Section 4, the control law $u(t)$ of the system (41) and (42) can be obtained as

$$
\begin{aligned}
u(t) & =-H_{1} \xi_{1}(t)-E_{2} \hat{y}(t)-H_{2} \xi_{2}(t)+u_{m}(t), \\
u_{m}(t) & =E_{3} \hat{r}_{m}(t)+H_{3} \xi_{3}(t) .
\end{aligned}
$$

The network topology is simple and is shown in Fig. 4. But this small scale network has been developed by using four computers, and some experiments have been done. This network consists of two senders with four data flows (this means four sessions and $N=8$ ), one bottleneck router and one receiver, which receives data from senders through the bottleneck router. The reference queue size is $\bar{q}_{0}=40$ [packets]. Thus $N=8$, $T_{p}=0.014, R_{0}=0.5742[s]$ and $D=71.4$ [packets $\left./ s\right]$ are calculated (Azuma et al., 2005a; 2005b;2005c). Then we have

$$
\begin{aligned}
\dot{x}(t)= & {\left[\begin{array}{cc}
-0.68 & 0 \\
13.93 & -1.74
\end{array}\right] x(t) } \\
& +\left[\begin{array}{c}
-22.87 \\
0
\end{array}\right] u\left(t-R_{0}\right), \\
y(t)= & {\left[\begin{array}{ll}
0 & 1
\end{array}\right] x(t) . }
\end{aligned}
$$

Assuming that $C_{m}=[2,0]$, we get $Q(p)=p+6$ and $T(p)=p-2.58$. The input $u(t)$ is given as

$$
\begin{aligned}
u(t)= & {\left[\begin{array}{ll}
36 & 6
\end{array}\right] \xi_{1}(t)-0.025 y\left(t+R_{0}\right) } \\
& +\left[\begin{array}{ll}
0.62 & 0.14
\end{array}\right] \xi_{2}(t)+u_{m}(t), \\
u_{m}(t)= & -0.078+\left[\begin{array}{ll}
0.32 & 0.054
\end{array}\right] \xi_{3}(t),
\end{aligned}
$$

where

$$
\begin{aligned}
& H_{1}=-\left[\begin{array}{ll}
36 & 6
\end{array}\right], \quad H_{2}=-\left[\begin{array}{ll}
0.62 & 0.14
\end{array}\right], \\
& H_{3}=\left[\begin{array}{ll}
0.32 & 0.054
\end{array}\right], \\
& E_{2}=0.025, \quad E_{3}=-0.078 .
\end{aligned}
$$

The output responses are shown in Fig. 5, and they converge to 40 [packets].

In this example, when the white noises $E \omega(t)=d(t)$ and $E_{0} \omega(t)=d_{0}(t)$ enter, the design of the congestion control system with time-delay is effective. The white noises are given as follows:

$$
\begin{aligned}
E \omega(t) & =0.6, & & 10 \leq t \leq 25, \\
E_{0} \omega(t) & =0.02-0.16 t, & & 38 \leq t \leq 55 .
\end{aligned}
$$

In the responses (see Fig. 6) of the congestion system with white noises, the output errors converge to zero. The effectiveness of this method has thus been verified. 


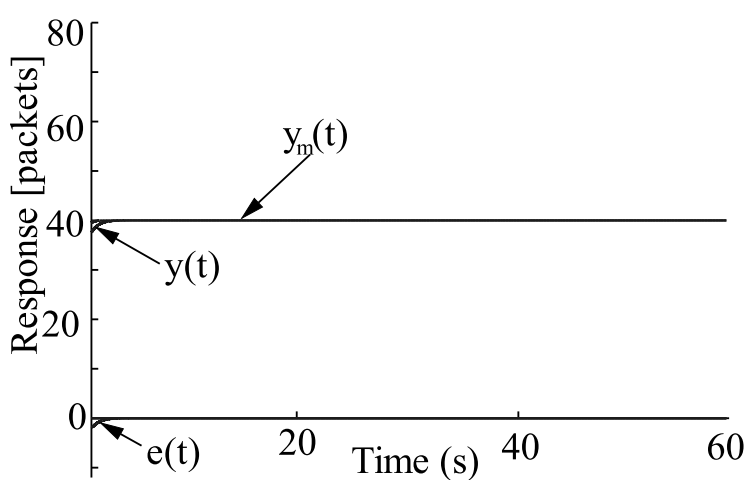

Fig. 5. Responses of the system with time-delay $\left(y_{m}(t)=\bar{q}_{0}\right)$.

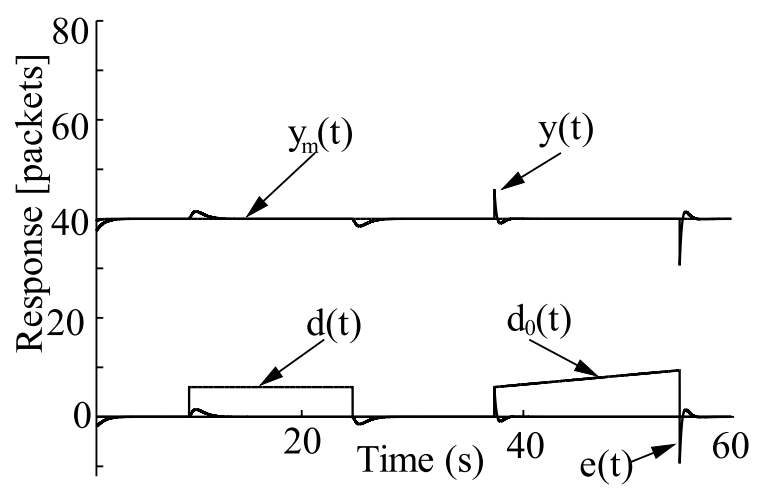

Fig. 6. Responses of the time-delay system with white noises $\left(y_{m}(t)=\bar{q}_{0}\right)$.

\section{Concluding remarks}

This paper studied a control system for state predictive with time-delay with a model following method which was one of the effective means of solving time-delay problems in a control system. The method could efficaciously control time-delay under disturbances and had excellent practicability.

By using state predictive control, the linear input time-delay system which describes a TCP/AQM network is transformed into an equivalent linear time invariant system, and it has been possible to design controllers based on linear control theories. For a congestion control problem, a round packet trip time is not stationary and depends on the actual traffic. (This is the case for the TCP protocol, and it is clearly given in the model (35).) From simulation results, it was verified that the congestion controller using the proposed approach is better.

\section{References}

Alevisakis, G. and Seborg, D. E. (1974). Control of multivariable systems containing time delays using a multivariable Smith predictor, Chemical Engineering Science 29(3): 373-380.

Akiyama, T., Hattori, H. and Okubo, S. (1998). Design of the model following control system with time delays, Transactions of the Institute of Electrical Engineers of Japan 118(4): 497-510.

Azuma, T., Fujita, T. and Fujita, M. (2005a). Congestion control for TCP/AQM networks using state predictive control, IEEE Transactions on Electronics, Information and Systems 125c(9): 1491-1496.

Azuma, T., Fujita, T. and Fujita, M. (2005b). A design of state predictive $H_{\infty}$ congestion controllers for TCP/AQM networks, Transactions of the Institute of Systems, Control and Information Engineers 18(10): 373-375.

Azuma, T., Naito, H. and Fujita, M. (2005c). Experimental verification of stabilizing congestion controllers using the network testbed, Proceedings of the 2005 American Control Conference, Portland, OR, USA, pp. 1841-1846.

Chiasson, J. and Loiseau, J. J. (2007). Applications of Time Delay Systems, Springer-Verlag, Berlin.

Gu, Y., Towsley, D., Hollot, C. V. and Zhang, H. (2007). Congestion control for small buffer high speed networks, Proceedings of IEEE Infocom, Anchorage, AK, USA.

Hollot, C. V., Misra, V., Towsley, D. and Gong, W. (2001a). On designing improved controllers for AQM routers supporting TCP flows, Proceedings of IEEE Infocom, Anchorage, AK, USA.

Hollot, C. V., Misra, V., Towsley, D. and Gong, W. (2001b). A control theoretic analysis of RED, Proceedings of IEEE Infocom, Anchorage, AK, USA.

Hollot, C. V., Misra, V., Towsley, D. and Gong, W. (2002). Analysis and design of controllers for AQM routers supporting TCP flows, IEEE Transactions on Automatic Control 47(6): 945-959.

Kim, B. K. and Bien, Z. (1981). Design of a time-optimal feedback controller for systems with delay in control, IEEE Transactions on Industrial Electronics and Control Instrumentation 28(1): 28-36.

Mascolo, S. (2000). Smith's principle for congestion control in high-speed daata networks, IEEE Transactions on Automatic Control 45(2): 358-364.

Misra, V., Gong, W. and Towsley, D. (2000). Fluid-based analysis of network of AQM routers supporting TCP flows with an aplication to RED, Proceedings of ACM SIGCOMM, Stockholm, Sweden.

Okubo, S. (1985). A design of nonlinear model following control system with disturbances, Transactions of the Society of Instrument and Control Engineers 21(8): 792-799.

Okubo, S. (1992). Nonlinear model following control system using stable zero assignment, Transactions of the Society of Instrument and Control Engineers 28(8): 939-946.

Watanabe, K. and Ito, M. (1981). A process-model control for linear system with delay, IEEE Transactions on Automatic Control 26(6): 1261-1269.

Wood, R. K. and Berry, M. W. (1973). Terminal composition control of a binary distillation control, Chemical Engineering Science 28(12): 1707-1717. 
Zhang, H. S. and Xie, L. H. (2007). Control and Estimation of Systems with Input/Output Delays, Springer-Verlag, Berlin.

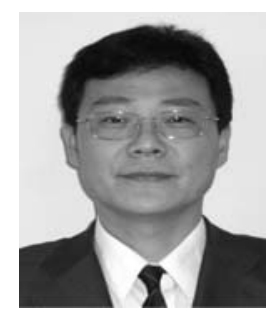

Dazhong Wang received the B.Eng. degree in chemical machines in 1990 from the Jilin Institute of Chemical Technology, P. R. China, the M.Eng. and Dr.Eng. degrees in automation in 2005 and 2008 from Yamagata University, Japan. Currently, he is a researcher in automation at Yamagata University. His research interests include automation and robotics, especially model following control systems theory, time-delay systems, neutral systems, descriptor systems, robust control, and robot control.

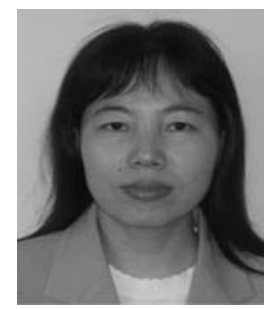

Shujing Wu received the B.Eng. degree in chemical machines in 1990 from the Jilin Institute of Chemical Technology, P. R. China, the M.Eng. degree in automation in 2006 from Yamagata University, Japan. Currently, she is a graduate in automation at Yamagata University, Japan. Her research interests include discrete time systems, descriptor systems, control theory of robots, networked control systems, and time-delay systems.

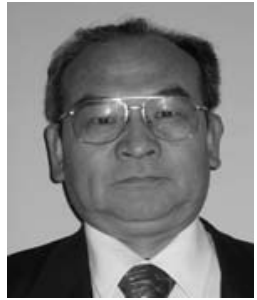

Shigenori Okubo received the B.Eng. degree in 1970 from Tohoku University, Japan. He obtained the M.Eng. and Dr.Eng. degrees from Tokyo University, Japan, in 1972 and 1975. $\mathrm{He}$ was a lecturer/associate professor at Tokyo University from 1980 to 1987 . He became an associate professor in 1988 and then a professor in 1990 at Yamagata University. He has published about 200 refereed journal and conference papers. Dr. Okubo is a member of the Society of Instrument and Control (Japan) and the Japan Society of Mechanical Engineers. His main research interests include the design of nonlinear control systems, time-delays systems, robot control, and model following control systems theory.

Received: 18 May 2008

Revised: 19 September 2008 